\title{
Shape conformable high spatial resolution tactile bracelet for detecting hand and wrist activity
}

\author{
Risto Kõiva and Eckard Riedenklau \\ Center of Excellence - \\ Cognitive Interaction Technology (CITEC), \\ Bielefeld University \\ D-33619 Bielefeld, Germany \\ e-mail: rkoiva|eriedenk@techfak.uni-bielefeld.de
}

\author{
Carla Viegas and Claudio Castellini \\ Robotics and Mechatronics Center \\ DLR - German Aerospace Center \\ D-82234 Weßling, Germany \\ e-mail: carlaluisa.deoliveiraviegas|claudio.castellini@dlr.de
}

\begin{abstract}
We present a novel sensory device that can noninvasively capture the muscle activations in the lower arm in unprecedented detail. The primary motivation for building the sensor was to have a new input channel to control modern stateof-the-art multi-degree-of-freedom prosthetic hands, but many interesting use cases have arisen such as its use as an input device in the areas of manual intelligence research, computer gaming and immersive virtual reality environments.
\end{abstract}

The modular compact tactile sensor bracelet has up to 320 highly sensitive sensor elements and measures the bulgings of muscles around the full circumference of the arm. In a preliminary experiment described in this paper, we trained a linear regression model to learn the mapping between the sensor values and flexion of three fingers plus two degrees of freedom of the wrist. The results show that the measured high dimensional force pattern corresponds to targeted single-digit activity.

The soft surface of the sensor and the flexible links between the single modules make the bracelet comfortable to wear and conformable to various arm and residual limb shapes.

\section{INTRODUCTION}

Our ability to physically interact with the world in large part is provided by our hands and therefore the loss of a hand is inevitably accompanied by a reduction in quality of life. In many cases, common low-cost hand prostheses can return some lost functionality, but they have many limitations not least of which is that most provide just a single degree-of-freedom (DOF) gripper. Recent advances in state-of-the-art multi-DOF hand prostheses are a significant first step towards providing hand amputees with dexterous capabilities. However, acceptance of these advances has been held back due to their awkwardness of use and their thus far non-reliable control [1]. Surface electromyography (sEMG) has historically been the main way that the hand movement intent of amputees was inferred [2]. By placing sEMG electrodes on top of the muscles of the lower arm responsible for actuating the fingers, a non-invasive method to gather muscle (and remnant muscle) activity is possible. However, as the lower arm contains a high number of finger driving muscles, and given that the muscles are closely packed side-by-side and sometimes even interwoven, invariably the resulting signal delivered by the sEMG electrodes is an extremely noisy mixture of multiple muscle activity. Even

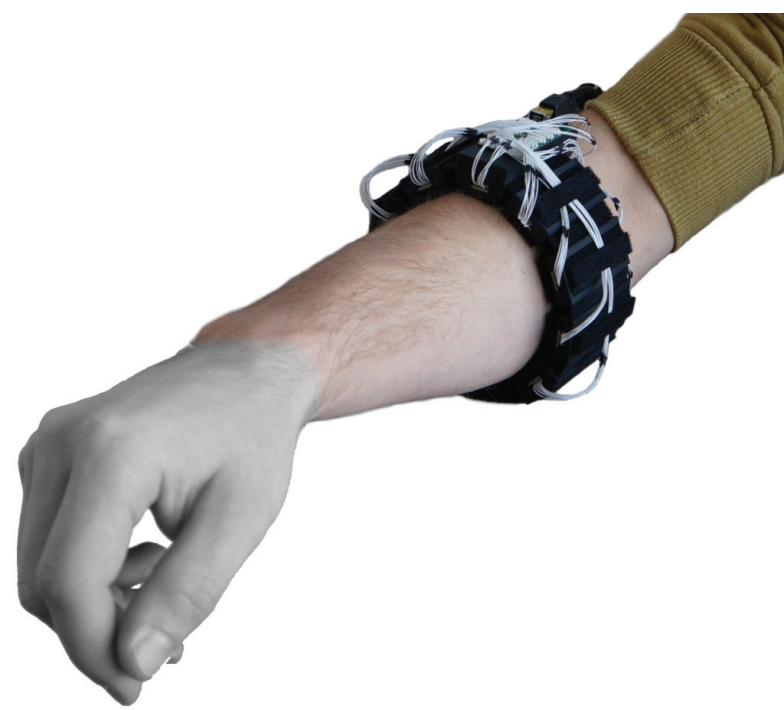

Fig. 1. A high-spatial resolution tactile bracelet with a soft and conformable shape is used to capture muscle activity in the lower forearm. One possible usage scenario for this bracelet would be to capture hand amputee intention in order to drive the single fingers of a modern multi-DOF hand prostheses.

when the latest state-of-the-art machine learning algorithms are used to interpret the data, robust high-fidelity hand activity intention detection is not yet possible, and this has led the assistive robotics community to call out for novel kinds of interfaces [3].

In our previous proof-of-concept work [4] we captured the muscle bulges using a flat tactile sensor array with high dynamic-range [5] from the ventral side of the arm only and using this data we were able to show that tactile sensing can lead to a very accurate description of lower arm muscle activity. The main idea stems from the fact that flexing a finger results in the thickening of the corresponding muscle, which in turn produces increased pressure on the surface of the skin above the location of the muscle. In this paper we extend our previous work by introducing a modular shape conformable bracelet based on a similar highly sensitive resistive tactile sensor technology to capture the muscle bulgings around the full circumference of the arm [Fig. 1]. 


\section{COMPARISON TO RELATED WORK}

The original idea of capturing muscle bulgings of residual limbs using force sensors was published as early as 1966 by Luccacini et al. [6]. At the time of the original experiments, high density tactile sensor technology was not yet available and simple bulky pressure sensitive binary switches were employed to detect actions. More recently, but in the same vein, Craelius et al. have explored the use of a hard socket fitted with 8 to 32 myo-pneumatic sensors [7] and 14 force-sensing resistors (FSRs) [8]. In both cases, they demonstrated that finger motion discrimination was possible. The same research group has recently applied the FSR-based approach (with 8 sensors) to gait control [9] and brain injury rehabilitation [10]. Gait control was also realized in this way in an earlier paper by Lukowicz et al. [11].

In a recent paper, Radmand et al. [12] presented a tactile cuff with $14 \times 9$ sensor cells for upper limb prosthetic control. The sensor had a spatial resolution of $10 \mathrm{~mm}$ and only the diameter of the otherwise solid cylindrical shape was adjustable. Yungher et al. [9] as well as Radmand et al. [12] were able to show that capturing surface muscle pressure yields a more stable and repeatable signal than common sEMG.

In this paper, we demonstrate the first soft and shape conformable solution in the form of a tactile bracelet able to capture upper limb muscle pressures in unprecedented detail.

\section{HARDWARE DESCRIPTION}

The tactile bracelet development builds upon our previous work [4] in which we used a flat resistive tactile sensor array by Schürmann et al. [5] to capture muscle bulgings of the forearm from the ventral side only. We now extend the original idea by developing a portable and shape conformable sensory device capable of capturing pressure information from around the full circumference of the arm [Fig. 1].

In line with the previously used flat tactile sensor array, the bracelet uses the same high performance resistive elastomer based tactile sensor technology, built upon the fact that the interface resistivity between two electrodes changes according to the applied load. The tactile sensor is made of a chemically golded Printed-Circuit-Board (PCB) surface as electrodes and a conductive elastomer foam as the sensor material, a technique first introduced by Weiss and Wörn [13]. The tactile sensor cell resistance, $R_{t}$, is the sum of three parts [see Fig. 2] - the variable surface interface resistance, consisting of $R_{s 1}+R_{s 2}$, and a constant sensor material volume resistance $R_{v}$. Using a voltage divider circuitry, we convert the variable sensor cell resistance into a voltage change, which we digitize with an analog-to-digital converter (ADC).

Fig. 3 shows the developed tactile sensor module with 32 electrodes in an $4 \times 8$ cell arrangement and embedded data acquisition electronics used to maximize the signal-tonoise ratio. The spatial resolution of the cells is $5 \mathrm{~mm}$. Two 16-channel 12-bit ADC's of type AD7490 digitize the tactile

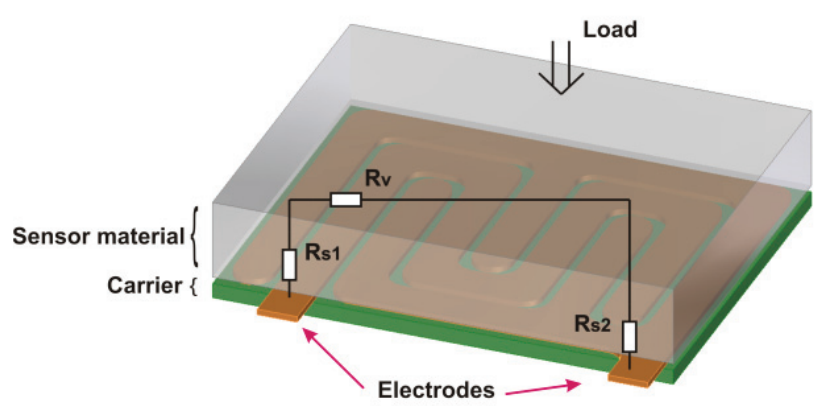

$R_{t}=R_{s} 1+R_{v}+R_{s} 2$

Fig. 2. The resistance of a single resistive tactile sensor cell, measured between two electrodes, is the sum of sensor material volume resistance and contact resistances between the sensor material and the electrodes. The contact resistance changes according to the applied load on the sensor foam.
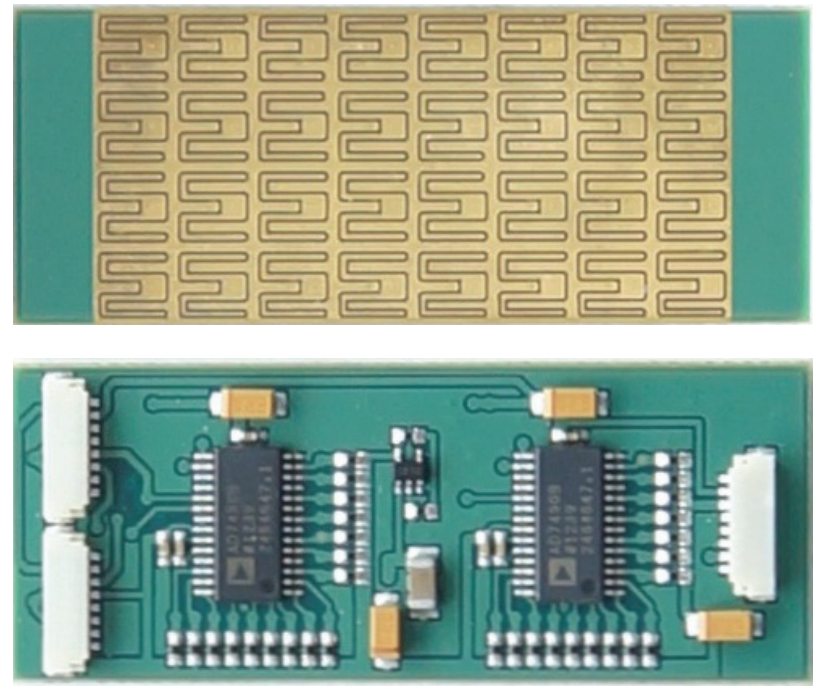

Fig. 3. A sensor module PCB with 32 tactile cells. (Top) The electrode side showing $4 \times 8$ arrangement of the M-shaped electrodes in a $5 \mathrm{~mm}$ grid. The non-conductive areas on the left and on the right of the electrode grid are reserved for attachment of the sensor elastomer with a double sided tape. (Bottom) The digitization circuitry is located directly on the backside to keep the analog signal path at a minimum.

cell values and forward the data onto an internal SPI-bus. Depending on the size and shape of the arm or residual limb, between seven and ten $20 \times 50 \mathrm{~mm}$ sized tactile sensor modules are usually required to capture force patterns from around the full circumference. The elastomer foam based resistive tactile sensors have a hyperbolic characteristic curve [13] that is very sensitive especially in the range of subtle to low forces that commonly occur from the muscle bulges in the forearm. Fig. 4 depicts the sensor output over 50 consecutive trials, in which we loaded the sensor from idle to $20 \mathrm{~N}$ on a measurement bench using a $1 \mathrm{~cm}^{2}$ probe tip. The graph shows the raw output of a single tactile sensor cell, as sampled using 12-Bit ADC (range 0 to 4095). When worn as a bracelet, the sensor output remained between idle and approximately $2500(\approx 60 \%$ of the full sensor range), which covers the initial linear portion of the hyperbolic sensor characteristic. 


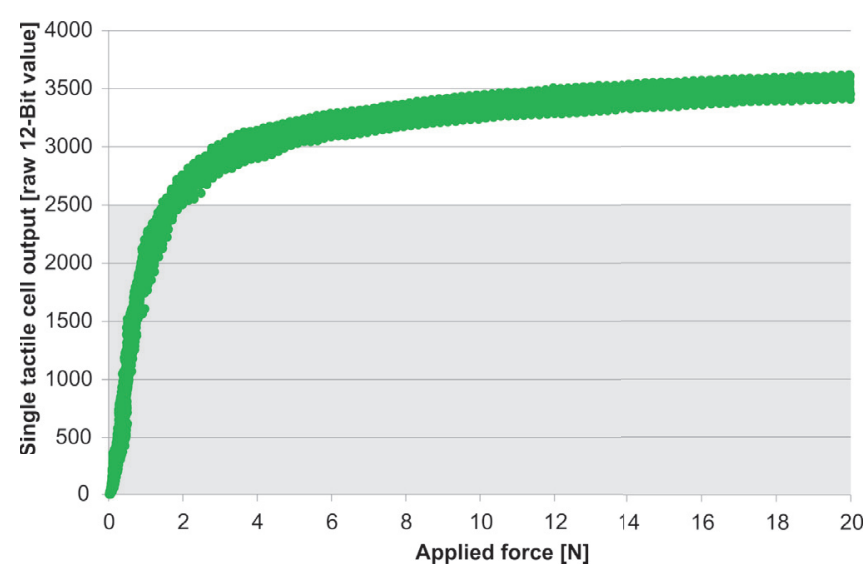

Fig. 4. Tactile sensor performance as measured using $1 \mathrm{~cm}^{2}$ circular probe tip over 50 trials. The sensor cell was loaded from idle to $20 \mathrm{~N}$ and the output measured over voltage divider circuit with an 12-bit ADC. As experiments revealed, for measuring muscle bulges of lower forearm, only the initial almost linear part of otherwise hyperbolic sensor characteristic is used (gray area).

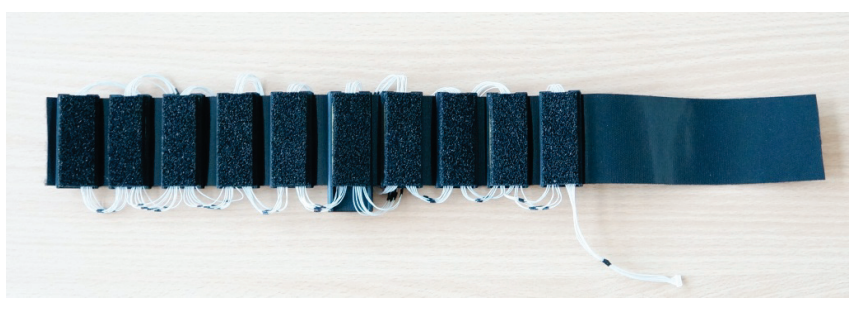

Fig. 5. Rolled out tactile bracelet shown from the side contacting the skin. The image displays the soft elastomer and the flexible hook-and-loop band for attachment. The amount and spacing of tactile sensor modules can be easily varied on the hook-and-loop band in order to accommodate different forearm sizes.

A wide flexible hook-and-loop band forms the basis of the bracelet, upon which the sensor modules are mounted with custom made plastic brackets. The brackets are designed to have high friction while attached to ensure minimum slippage. On the other hand, when rolled out flat, as shown in Fig. 5, modules can be added, removed or repositioned with little effort. Similar to a blood pressure measurement device, the hook-and-loop band makes it easy to adjust the tension of the bracelet around the arm.

A single data arbitration board with a PIC32MX microcontroller, running at $80 \mathrm{MHz}$, gathers the data from tactile sensor modules over the single daisy-chained SPI-bus and streams it out over a USB bus using a virtual serial port (USB-CDC). This enables effortless connectivity to a wide variety of existing data analysis software. The microcontroller firmware, developed using a Microchip C32 compiler, samples the connected ADCs in a round-robin fashion. Such a sampling scheme maximizes the time available after the ADC channel selection, which indirectly improves the signal-to-noise ratio of the measurements. The acquisition circuitry achieves a sampling speed of approximately 88 frames-per-second with 10 connected tactile modules (320 tactile cells). A second plastic bracket type was developed to mount the data arbitration board in a very compact way onto one sensor module. Fig. 6 illustrates the electrical SPI

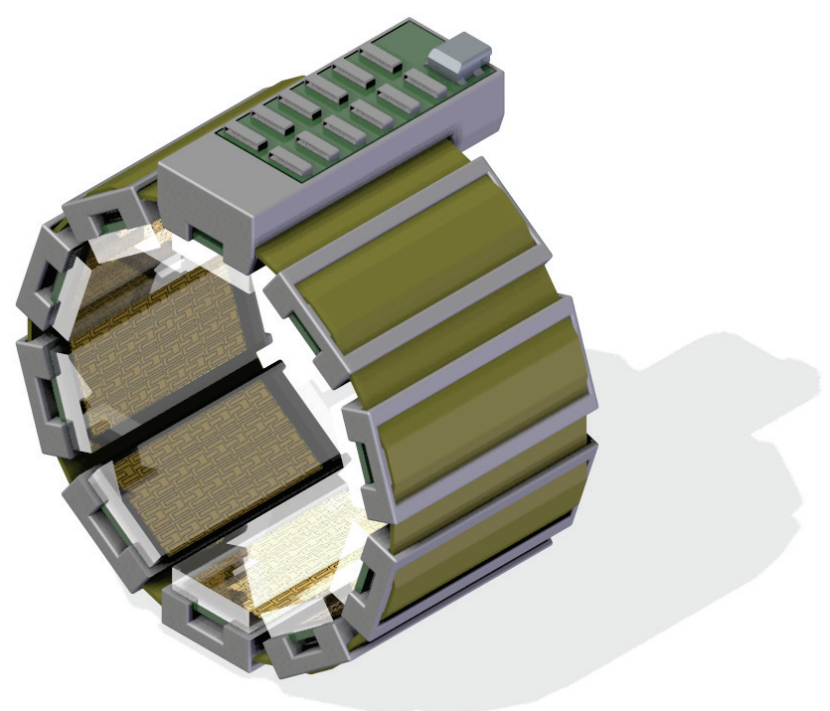

Fig. 7. A CAD sketch of the modular bracelet with the conductive tactile sensor elastomer foam rendered translucent, allowing us to observe the underlying sensor electrodes.

bus and power distribution routing of the tactile bracelet. Special care was taken to achieve stable power supply voltage for the ADC's by implementing a $5 \mathrm{~V}$-in/5V-out DC/DC converter to filter the USB supply voltage. The bracelet's data arbitration board carries an InvenSense MPU9150 9-axis Inertial-Measurement-Unit, making it possible to capture the arm acceleration, orientation relative to earth's magnetic north and orientation relative to ground.

Fig. 7 displays the rendering of the tactile bracelet with 10 sensor modules, depicting the soft, normally black, conductive elastomer foam in translucent for an improved view of the sensor electrodes.

\section{EXPERIMENT DESCRIPTION}

A preliminary experiment was conducted to investigate whether the measured muscle bulgings can not only be used to identify different finger flexions, but also distinct wrist movements. For this purpose, data from participants was recorded while they tried to mimic various demonstrated actions. Custom software was developed to visualize a 3D simulated hand model as a stimulus for participants, to sequence the stimulus movements and to record and visualize the tactile sensor intensities.

\section{A. Participants and experiment protocol}

The experiment was performed in accordance with the Declaration of Helsinki. Three intact participants aged between 22 and 40 years participated in the trials. The procedure was thoroughly explained before the onset of the trial and, if required, assistance with their understanding of the stimulus was provided during the trial.

Each participant was asked to sit comfortably on an office chair in front of a monitor. The tactile bracelet was fitted directly onto the skin on the right upper forearm in a similar 


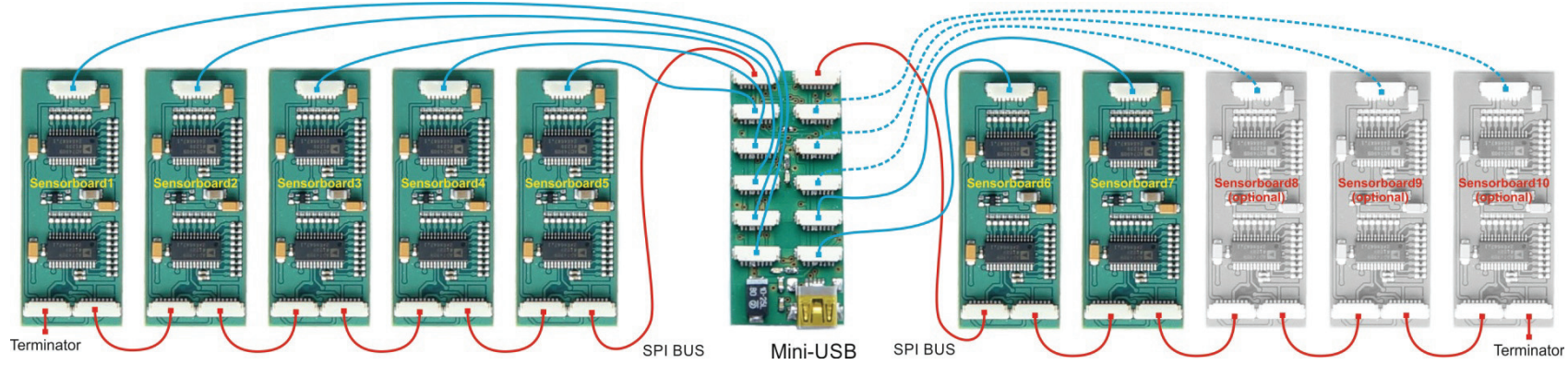

Fig. 6. Tactile bracelet's electrical routing. A single data arbitration board with a PIC32MX microcontroller (in the middle of the image) connects via a single daisy-chained SPI bus (depicted in red) to up to 10 sensor modules. Dedicated power distribution and addressing lines (depicted in blue) run from the data arbitration board to each sensor module. The data arbitration board streams out the collected tactile sensor data using the USB-CDC serial stream protocol.

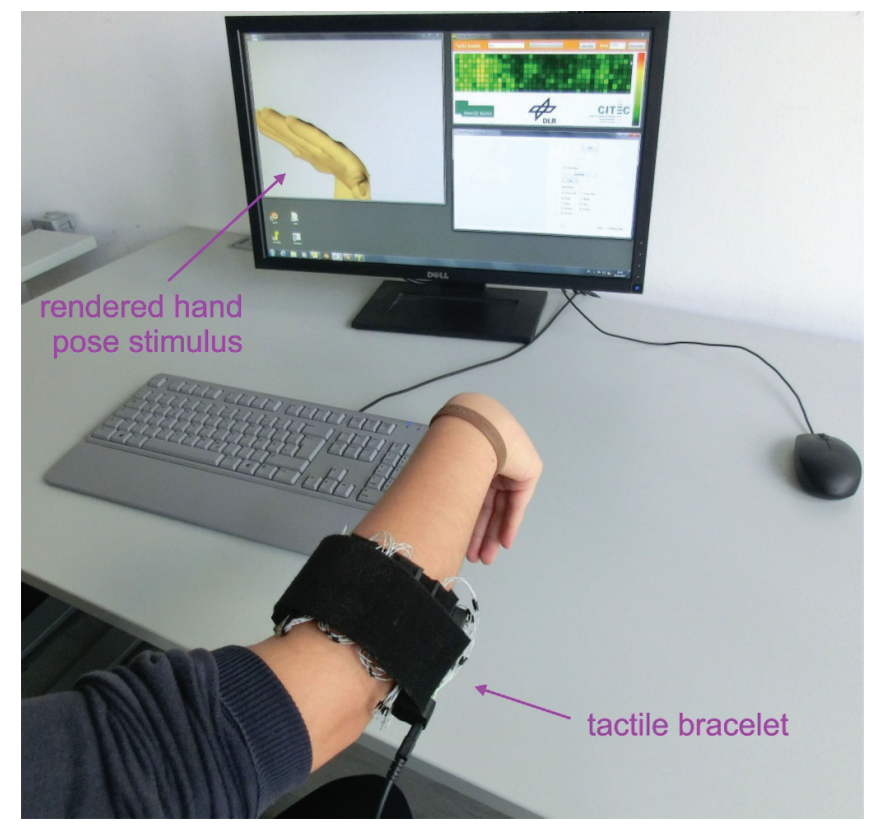

Fig. 8. A bird's-eye view of the experimental setup. Participants, wearing the tactile bracelet around the lower forearm, tried to match the pose of the $3 \mathrm{D}$ rendered hand presented on the monitor.

orientation for all participants. The boards numbered 1-4 were placed on the ventral side of the forearm, where the main flexor muscles are located and the boards numbered 5-10 were placed on the dorsal side of the forearm, upon the extensor muscles.

Once data collection began, the participants were asked to imitate the movements of a simulated hand model which was displayed on the monitor [Fig. 8]. The sequence of movements they were presented with consisted of six different movements: thumb rotation, flexion of the index and little finger, wrist flexion, extension and supination. Each participant repeated this sequence of movements ten times. Finger flexions were executed by pressing the fingers against the table; wrist motions were executed "in the air". The complete experiment, including the consent and the introduction to the exercises, lasted approximately 15 minutes.

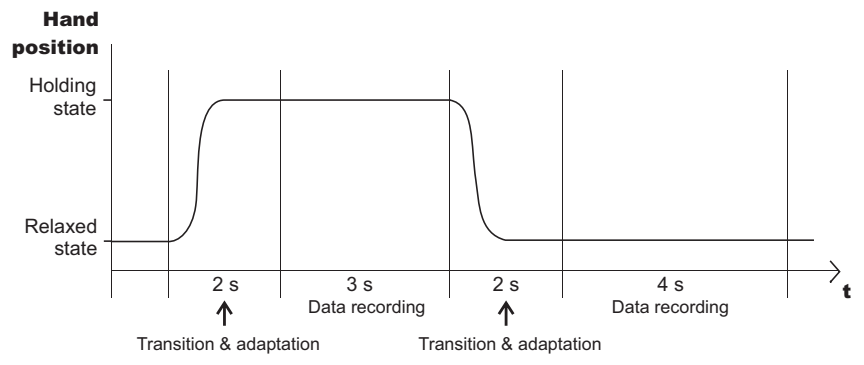

Fig. 9. Timing of a single experiment cycle depicting the hand movement state transitions and data recording intervals. After each stimulus state transition, participants were given two seconds to react to the new stimulus and for the movement towards this stimulus to stabilize, before the data capture of the state began.

\section{B. Data acquisition and processing}

Fig. 9 depicts the timing of the hand model stimulus used. Each cycle lasted 10 seconds and consisted of a movement holding state and a relaxed state. After each state transition, the participants were given $2 \mathrm{~s}$ to perceive and realize the necessary movement before the data collection was started. Data was collected for $3 \mathrm{~s}$ for each exercise and for $4 \mathrm{~s}$ for the resting position.

Data acquired from the tactile bracelet and the state of the 3D rendered hand stimulus were synchronized by linearly interpolating the timestamps of the respective data channels. The tactile sensor data was bandpass filtered using a firstorder Butterworth filter with cutoff frequencies at 0.01 and $1 \mathrm{~Hz}$ in order to remove high-frequency disturbance as well as long term signal drift due to elastomer viscosity and humidity changes (e.g. due to sweating).

\section{EXPERIMENTAL RESULTS}

Fig. 10 shows the average activation tactile patterns obtained for each participant and each movement. Each pattern was obtained by averaging over the 10 repetitions of the "holding" state and subtracting the averaged relaxed state values of the bracelet values for each movement [see Fig. 9]. This was done in order to have a differential view of the patterns, as well as to eliminate any possible undesired effect of hysteresis and/or the time required for the participant to actually reach the "holding" state. Each pattern was then 

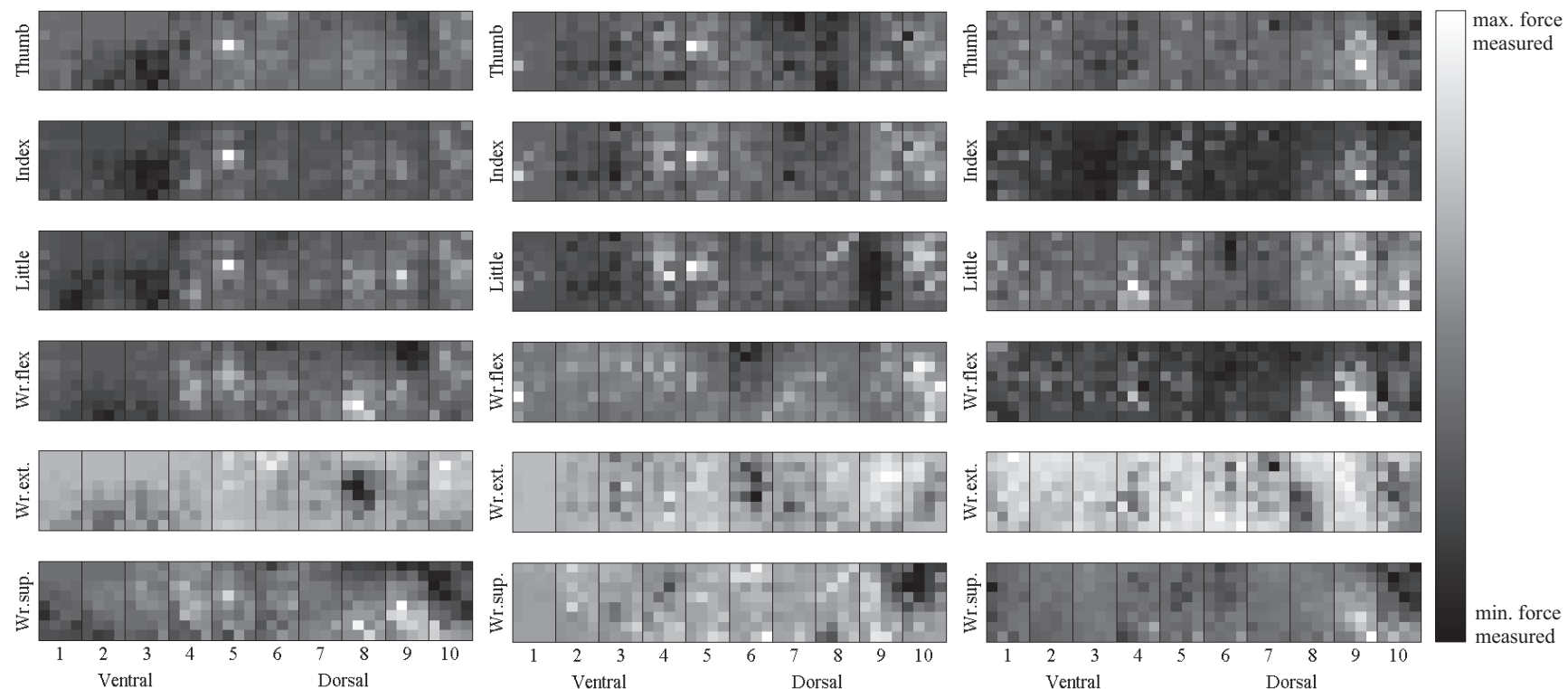

Fig. 10. Typical tactile patterns for each participant (left, center, right) and movement. Tactile modules 1-4 were positioned on the ventral part of the forearm, while tactile modules 5-10 covered the dorsal part. Each pattern is shown with separately normalized gray-scale coding between the lowest (black) and highest (white) force sampled. A muscle bulge corresponding to higher force on the sensor is depicted in lighter color and the areas that produced less force on the sensor during activity are depicted in darker color.

TABLE I. REgression Normalized Root-MeAn-SqUare ERror [\%] FOR EACH PARTicipant AND PERFormed MOVEMENT.

\begin{tabular}{c|ccc|ccc}
\hline Participant & Thumb & Index & Little & Wr.flex. & Wr.ext. & Wr.sup. \\
\hline$\# 1$ & $15.22 \pm 0.3$ & $11.56 \pm 0.2$ & $11.30 \pm 0.3$ & $9.52 \pm 0.2$ & $9.81 \pm 0.3$ & $11.28 \pm 0.2$ \\
$\# 2$ & $15.02 \pm 0.4$ & $12.14 \pm 0.3$ & $11.03 \pm 0.2$ & $11.31 \pm 0.3$ & $10.00 \pm 0.2$ & $10.21 \pm 0.3$ \\
$\# 3$ & $12.05 \pm 0.2$ & $11.63 \pm 0.3$ & $11.62 \pm 0.2$ & $9.82 \pm 0.2$ & $8.60 \pm 0.3$ & $9.48 \pm 0.2$ \\
\hline
\end{tabular}

normalized to values between 0 and 1 (gray-scale coded in Fig. 10 between black and white).

Clear similarities can be observed among the fingerflexion patterns and the wrist flexion for each single subject. This is very likely due to the similar usage of the flexor muscles in these kinds of movements. Notice the activation on the right-hand side of the wrist flexion patterns (boards $8-10$ ), at the border between the flexor and extensors region, also coherent with the human anatomy. The wrist extensions show, as was to be expected, increased activity on the dorsal side (boards 6-9), as the extensor muscles are used for this movement. Lastly, a remarkably localized "double-sided" pattern, with high and low values close together, appears in the wrist supination patterns on the dorsal side (boards 8-10), denoting the activation of the M. Brachioradialis, involved in wrist pronation/supination. As far as inter-subject analogy is concerned, the patterns depicted in Fig. 10 only show some degree of agreement, although in this case the per-movement normalization tends to weaken the visually observable similarity.

Table I shows the results of applying linear regression (in the regularized form of Ridge Regression (RR), see, e.g. [14]) in order to predict the visual stimulus values from the tactile bracelet. In this case, cross-validation was applied to obtain a statistically significant estimation. The entire dataset for each participant and movement was randomly shuffled, then one tenth of it was used to train the RR and the test was performed on the remaining $90 \%$. This procedure was repeated for 50 times with a different random shuffle each time. The Normalized Root-Mean-Square-Error (NRMSE) was then evaluated between the actual stimulus values and the values predicted by RR. Table I shows the average plus/minus one standard deviation of the NRMSE values over the 50 trials.

As we can see, the standard deviation is always remarkably smaller with respect to the average values, denoting a high stability in the results. The NRMSE values range from about $9.5 \%$ to $15 \%$. This is in line with the accuracy values found elsewhere using different approaches; for instance, see [15], where sEMG, pressure sensing and ultrasound imaging were compared on a similar problem. Of the three approaches, pressure sensing performed best with an NRMSE of between $4 \%$ and $14 \%$.

We note that the error values for the thumb rotation are slightly higher than the other movements. This is to be expected, since the majority of muscles involved in thumb movement are found inside or close to the hand which is not directly measured by our bracelet. Lastly, we note that the movements involving the wrist are in general better predicted than the single-finger movements as they required a larger involvement of muscles in the forearm.

\section{COnClusions And Future Work}

In this paper we introduced a novel high spatial resolution tactile bracelet that can be used to non-invasively capture the hand and wrist activity by observing muscle 
bulgings in the lower arm. Up to 320 highly sensitive tactile cells, in a dense $5 \mathrm{~mm}$ spatial resolution, provide an unprecedented insight into lower arm muscle activity. Soft conductive elastomer material combined with a shape conformable modular design make the bracelet comfortable to wear and its adaptability allows it to fit a wide variety of arm or residual limb shapes and sizes. A sampling speed of close to $100 \mathrm{~Hz}$ provides a high fidelity account of muscle activity, and USB connectivity (serial data streaming based on the CDC protocol) eliminates the necessity of system specific drivers.

As the focus of this paper was to present the bracelet itself, the experimental results are to be treated as preliminary. Nevertheless, the results proved to be highly promising. Three able bodied participants engaged in repetitive finger flexions and wrist movements that are crucial to the control of a hand prosthesis. A qualitative examination of the tactile patterns corresponding to each movement revealed the ability of the bracelet to capture the bulging of the muscles involved in each of the movements. Furthermore, a very simple machine learning regression method, applied directly to the bandpass-filtered tactile values, was able to predict the type of movement and its intensity with a normalized error which is in line with previous approaches.

We claim that tactile sensing represents a new frontier for intent detection for amputees. The tactile bracelet, together with a slightly more refined machine learning / feature extraction system than the one showed here, could potentially represent an optimal human-machine interface for the disabled, to be used in rehabilitation in a virtual environment, as well as to directly control hand prosthetics.

The developed tactile bracelet has great potential to provide interesting use cases for other research fields as well, such as manual intelligence research, computer gaming and immersive virtual reality environments.

\section{ACKNOWLEDGMENTS}

The authors would like to thank Vitali Pojurov and Tobias Röhlig of Bielefeld University for helping with the PCB routing and with porting the driver software to Linux. We would also like to thank Jonathan Maycock for his help proof-reading the manuscript. This work was partially supported by the DFG Center of Excellence EXC 277: Cognitive Interaction Technology (CITEC).

\section{REFERENCES}

[1] B. Peerdeman, D. Boere, H. Witteveen, R. Huis in 't Veld, H. Hermens, S. Stramigioli, H. Rietman, P. Veltink, and S. Misra, "Myoelectric forearm prostheses: State of the art from a usercentered perspective," Journal of Rehabilitation Research \& Development (JRRD), vol. 48, no. 6, pp. 719-738, Aug. 2011. [Online]. Available: http://dx.doi.org/10.1682/JRRD.2010.08.0161

[2] S. Micera, J. Carpaneto, and S. Raspopovic, "Control of hand prostheses using peripheral information," IEEE Reviews in Biomedical Engineering (R-BME), vol. 3, pp. 48-68, 2010.

[3] N. Jiang, S. Dosen, K.-R. Müller, and D. Farina, "Myoelectric control of artificial limbs - is there a need to change focus?" IEEE Signal Processing Magazine, vol. 29, no. 5, pp. 148-152, 2012.

[4] C. Castellini and R. Kõiva, "Using a high spatial resolution tactile sensor for intention detection," in 13th International Conference on Rehabilitation Robotics (ICORR), Seattle, Washington, USA, 2013.

[5] C. Schürmann, R. Kõiva, R. Haschke, and H. Ritter, "A modular high-speed tactile sensor for human manipulation research," in IEEE World Haptics Conference (WHC), Istanbul, Turkey, June 2011, pp. 339-344.

[6] L. F. Lucaccini, P. K. Kaiser, and J. Lyman, "The French electric hand: Some observations and conclusions," Bulletin of Prosthetics Research, vol. 10, no. 6, pp. 31-51, 1966.

[7] D. J. Curcie, J. A. Flint, and W. Craelius, "Biomimetic finger control by filtering of distributed forelimb pressures," IEEE Transactions on Neural Systems and Rehabilitation Engineering (TNSRE), vol. 9, no. 1, pp. 69-75, 2001. [Online]. Available: http://dx.doi.org/10.1109/7333.918278

[8] M. Wininger, N. Kim, and W. Craelius, "Pressure signature of forearm as predictor of grip force," Journal of Rehabilitation Research and Development (JRRD), vol. 45, no. 6, pp. 883-892, 2008.

[9] D. A. Yungher, M. T. Wininger, J. Barr, W. Craelius, and A. J. Threlkeld, "Surface muscle pressure as a measure of active and passive behavior of muscles during gait," Medical Engineering \& Physics, vol. 33, no. 4, pp. 464-471, 2011. [Online]. Available: http://dx.doi.org/10.1016/j.medengphy.2010.11.012

[10] D. Yungher and W. Craelius, "Improving fine motor function after brain injury using gesture recognition biofeedback," Disability and Rehabilitation: Assistive Technology, vol. 7, no. 6, pp. 464-468, 2012. [Online]. Available: http://dx.doi.org/10.3109/17483107.2011. 650782

[11] P. Lukowicz, F. Hanser, C. Szubski, and W. Schobersberger, "Detecting and interpreting muscle activity with wearable force sensors," in Pervasive Computing, ser. Lecture Notes in Computer Science, K. P. Fishkin, B. Schiele, P. Nixon, and A. Quigley, Eds. Springer Berlin Heidelberg, 2006, vol. 3968, pp. 101-116. [Online]. Available: http://dx.doi.org/10.1007/11748625_7

[12] A. Radmand, E. Scheme, and K. Englehart, "High resolution muscle pressure mapping for upper limb prosthetic control," in University of New Brunswick's Myoelectric Controls/Powered Prosthetics Symposium (MEC), Fredericton, New Brunswick, Canada, Aug. 2014, pp. 189-193. [Online]. Available: http://www. unb.ca/conferences/mec/_resources/docs/mec14_proceedings.pdf

[13] K. Weiss and H. Wörn, "The working principle of resistive tactile sensor cells," in IEEE International Conference Mechatronics and Automation (ICMA), vol. 1, Niagara Falls, Canada, July 2005, pp. 471-476.

[14] A. E. Hoerl and R. W. Kennard, "Ridge regression: Biased estimation for nonorthogonal problems," Technometrics, vol. 12, pp. 55-67, 1970.

[15] V. Ravindra and C. Castellini, "A comparative analysis of three noninvasive human-machine interfaces for the disabled," Frontiers in Neurorobotics, vol. 8, no. 24, 2014. 